RESPIRATORY INFECTION

\title{
Comparison between pathogen directed antibiotic treatment and empirical broad spectrum antibiotic treatment in patients with community acquired pneumonia: a prospective randomised study
}

\author{
M M van der Eerden, F Vlaspolder, C S de Graaff, T Groot, W Bronsveld, H M Jansen, \\ W G Boersma
}

Thorax 2005;60:672-678. doi: 10.1136/thx.2004.030411

See end of article for authors' affiliations

Correspondence to: Dr W G Boersma Department of Pulmonary Diseases, Medical Centre Alkmaar, Wilhelminalaan $12,1815 \mathrm{JD}$, Alkmaar, the Netherlands; w.boersma@ mca.nl

Received 21 June 2004

Accepted 21 April 2005

\begin{abstract}
Background: There is much controversy about the ideal approach to the management of community acquired pneumonia (CAP). Recommendations differ from a pathogen directed approach to an empirical strategy with broad spectrum antibiotics.

Methods: In a prospective randomised open study performed between 1998 and 2000, a pathogen directed treatment (PDT) approach was compared with an empirical broad spectrum antibiotic treatment (EAT) strategy according to the ATS guidelines of 1993 in 262 hospitalised patients with CAP. Clinical efficacy was primarily determined by the length of hospital stay (LOS). Secondary outcome parameters for clinical efficacy were assessment of therapeutic failure on antibiotics, 30 day mortality, duration of antibiotic treatment, resolution of fever, side effects, and quality of life.

Results: Three hundred and three patients were enrolled in the study; 41 were excluded, leaving 262 with results available for analysis. No significant differences were found between the two treatment groups in LOS, 30 day mortality, clinical failure, or resolution of fever. Side effects, although they did not have a significant influence on the outcome parameters, occurred more frequently in patients in the EAT group than in those in the PDT group $(60 \% v 17 \%, 95 \% \mathrm{Cl}-0.5$ to $-0.3 ; \mathrm{p}<0.001)$.

Conclusions: An EAT strategy with broad spectrum antibiotics for the management of hospitalised patients with CAP has comparable clinical efficacy to a PDT approach.
\end{abstract}

C ommunity acquired pneumonia (CAP) remains a common and serious illness. ${ }^{1}$ In the past decade several guidelines concerning the management of CAP have been published and recently revised by different organisations. ${ }^{2-5}$ However, recommendations range from a pathogen directed approach to an empirical strategy with broad spectrum antibiotics. The main advantage of pathogen directed treatment is the careful use of antibiotics, theoretically resulting in a reduction in adverse events and in antimicrobial resistance. ${ }^{67}$ Moreover, by performing microbiological investigations epidemiologically, important organisms such as Legionella pneumophila, drug resistant Streptococcus pneumoniae, and methicillin resistant Staphylococcus aureus may be detected. The main argument against a pathogen directed approach is the lack of sensitivity and specificity of the routine diagnostic methods currently employed. ${ }^{489}$ For this reason, sputum examination by Gram stain and culture were not recommended in the American Thoracic Society (ATS) guidelines as an aid to directing initial treatment. ${ }^{34}$

Interestingly, the guidelines are primarily consensus based. Up to now these guidelines have not been prospectively validated and compared with other therapeutic regimen(s) in a clinical setting. We therefore performed a prospective randomised study in which a pathogen directed approach was compared with empirical treatment consisting of broad spectrum antibiotics according to the ATS guidelines of 1993 in hospitalised patients with CAP. New ATS guidelines were presented in 2001 but were essentially the same as those of 1993 with the exception of treatment of patients in the intensive care unit (ICU). In both ATS guidelines the use of empirical broad spectrum antibiotics was emphasised.
The main outcome parameter in this study was the assessment of length of hospital stay (LOS); secondary outcome measures were therapeutic failure on antibiotic treatment, 30 day mortality, duration of antibiotic treatment, resolution of fever, adverse events, and quality of life.

\section{METHODS \\ Patients}

A prospective randomised study was performed between December 1998 and November 2000 in the Departments of Pulmonary Diseases and Internal Medicine at the Medical Centre Alkmaar, a teaching hospital with 900 beds. The medical ethics committee of the hospital approved the study. Patients who fulfilled the following criteria were enrolled in the study after giving written informed consent: (1) age 18 years or over; (2) clinical presentation of an acute illness with one or more of the following symptoms suggesting CAP: presence of fever $\left(\geqslant 38.0^{\circ} \mathrm{C}\right)$, dyspnoea, coughing (with or without expectoration of sputum), chest pain; (3) presence of new consolidation(s) on the chest radiograph. Patients were excluded from the study if one of the following criteria applied: presence of severe immunosuppression (HIV infection, high dose of immunosuppressive agents such as prednisone $>35 \mathrm{mg} /$ day, chemotherapy); presence of malignancy; pregnancy or breast feeding; documented severe allergy to antibiotics; presence of obstruction pneumonia; pneumonia within 8 days of hospital discharge.

Abbreviations: CAP, community acquired pneumonia; EAT, empirical broad spectrum antibiotic treatment; PDT, pathogen directed treatment 
As is common in our healthcare system, most of the patients first consulted their general practitioner (GP) and from this population a selected group was referred to our hospital. An assessment of disease severity was performed on admission using the pneumonia severity index (PSI) by one of the investigators (MMvdE) ${ }^{10}$ The treating physician was not aware of the PSI risk class stratification.

\section{Study design}

The patients were randomised on admission to one of the two treatment groups by means of cards in sealed envelopes. The empirical antibiotic treatment (EAT) group received antibiotic treatment according to the ATS guidelines of $1993 .{ }^{3}$ Beta-lactam $/ \beta$-lactamase inhibitor plus erythromycin were given intravenously (IV). Ceftazidime and erythromycin IV were given to patients referred to the ICU. Physicians were blind to the outcome of microbial studies. The microbiologist reported the results to the treating physician in case of failure on antibiotic treatment or when a pathogen was identified that was inadequately covered according to microbial investigation by the empirical regimen.

The pathogen directed treatment (PDT) group received IV treatment directed at the pathogen suspected to be the causative agent, as reported from routine microbial investigation or from clinical presentation (table 1). The results of a Gram stain (presence of $>25$ polymorphonuclear leucocytes and $<10$ squamous cells at $100 \times$ magnification) from sputum or pleural fluid, pneumococcal antigen detection (latex agglutination; Murex Diagnostics, Dartford, UK) in sputum or pleural fluid, and L pneumophila serogroup 1 urinary antigen detection test (enzyme immunoassay, BinaxNOW, Binax, Portland, Maine, USA) could be obtained within 2 hours of admission 24 hours a day.

\section{Outcome}

Clinical efficacy was primarily determined by the assessment of LOS. No criteria for discharge were given to the treating physicians, nor were they informed about the contribution of LOS as an outcome parameter for this study. The investigator was not actively involved in nor interfered with the treatment of any of the patients.

Secondary outcome parameters included therapeutic failure on antibiotic treatment. Clinical failures were subdivided into patients showing an early failure after adequate or inadequate treatment and patients showing a late failure on antibiotic treatment. An early failure was defined as patients in whom signs and symptoms of pneumonia did not improve within 72 hours of antibiotic treatment and persisted or progressed thereafter. A late failure was defined as a patient in whom signs and symptoms of pneumonia returned after 72 hours of admission following an initially good response on antibiotic treatment or those who died before the 30 day follow up. When, in the case of a failure, no pathogen had been identified or when the subsequently isolated pathogen had not been appropriately treated (according to microbial results and antibiotic sensitivity patterns) from the start of treatment, this was termed a failure on inadequate treatment. Other secondary outcome parameters included 30 day mortality, duration of intravenous antibiotic treatment, total duration of antibiotic treatment, resolution of fever, presence of adverse events, and quality of life. Adverse events were reported by the treating physician as symptoms or signs that could not be attributed to the pneumonia after starting antibiotic treatment. A quality of life analysis was performed using the Short-Form 36 Health Survey (SF-36) scale ${ }^{11}$ and CAP Symptom Severity score (SSC). ${ }^{12}$ The SF-36 questionnaire was administered during treatment and at the follow up visits 1, 3, and 6 months after treatment. A higher SF-36 score reflected a higher sense of quality of life. The SSC included the presence of five symptoms (cough, dyspnoea, sputum production, pleuritic chest pain, and fatigue). The severity of each symptom was scored on a 6-point scale (from $0=$ not present to $5=$ severe). The SSC was calculated on days 1 and 7 of treatment and at follow up 1, 3, and 6 months after treatment. A lower SSC indicated a better quality of life. The SF-36 and SSC scores were calculated according to the method described by Metlay et al. ${ }^{12}$

\section{Microbiological investigations}

For both groups a sputum specimen was obtained at admission for Gram staining, semi-quantitative culture, and $S$ pneumoniae antigen detection testing; three sets of blood cultures were taken and, if clinical symptoms suggested, a urine sample for $L$ pneumophila serogroup 1 antigen detection was obtained at admission, preferably before the start of antibiotic treatment. A bronchoalveolar lavage (BAL) specimen and protected specimen brush (PSB) with Gram stain, semi-quantitative culture, and $S$ pneumoniae antigen detection were performed, after consent, when patients did not expectorate sputum within 24 hours of admission or in case of clinical failure. Thoracentesis with Gram staining, $S$ pneumoniae antigen detection, and culture for aerobic and anaerobic bacteria was performed when pleural fluid was present. Blood samples for serology (Serion ELISA classic, Virion GmbH, Würzburg, Germany) were obtained on days 1 and 14 of treatment for the detection of antibodies to Mycoplasma pneumoniae, Chlamydia pneumoniae, L pneumophila serogroup 1-7, influenza A and B virus, parainfluenza virus $1-3$, respiratory syncytial virus (RSV), and adenovirus. After completing the study, all patients were analysed for the presence of $S$ pneumoniae antigen (enzyme immunoassay, Binax-NOW, Binax, Portland, Maine, USA) in the urine samples obtained at admission.

\begin{tabular}{lll}
$\begin{array}{l}\text { Table } 1 \text { Recommendations for antibiotic treatment directed at clinical presentation in } \\
\text { pathogen directed treatment group }\end{array}$ & Suspected pathogen & $\begin{array}{l}\text { Recommended antibiotic } \\
\text { treatment }\end{array}$ \\
\hline Clinical presentation & Streptococcus pneumoniae & Penicillin \\
\hline $\begin{array}{l}\text { Acute illness, lobar infiltrate, raised WBC } \\
\text { with an increase in PMNs }\end{array}$ & Atypical bacterial pathogen* & Erythromycin \\
$\begin{array}{l}\text { Mild illness, headache, upper airway tract } \\
\text { symptoms, young age, travel to southern } \\
\text { Eorope, contact with animals }\end{array}$ & $\begin{array}{l}\text { Spneumoniae, Haemophilus } \\
\text { influenzae, Moraxella } \\
\text { catarrhalis, Gram negative } \\
\text { Enterobacteriaceae, anaerobes } \\
\text { Staphylococcus aureus }\end{array}$ & Amoxicillin-clavulanate \\
& Flucloxacillin \pm gentamicin \\
\hline Influenza epidemic & &
\end{tabular}

WBC, white blood cells; PMN, polymorphonuclear leucocytes.

*Mycoplasma pneumoniae, Legionella pneumophila, Chlamydia pneumoniae/psitacci. 
Definitive aetiology was defined as (1) identification of an aetiological agent in the blood and/or pleural fluid; (2) detection of L pneumophila spp antigen in the urine; (3) the presence of $S$ pneumoniae antigen in pleural fluid; and (4) a threefold increase in antibody titre of $L$ pneumophila serogroup 1-7, M pneumoniae, C pneumoniae, influenza A and $\mathrm{B}$, parainfluenza virus $1-3, \mathrm{RSV}$ or adenovirus according to the manufacturer. Presumptive aetiology was defined as ( 1 ) a positive sputum, BAL or PSB culture by semi-quantitative methods compatible with the organism(s) seen on a good Gram stain specimen, (2) detection of $S$ pneumoniae antigen in urine or sputum, BAL or PSB specimen; (3) a single raised IgM titre of $\geqslant 17 \mathrm{U} / \mathrm{ml}$ for $M$ pneumoniae.

\section{Statistical analysis}

The patient sample size was calculated from the following assumptions: a reduction in LOS for patients with CAP by 3.4 days $(23 \%)$ following implementation of EAT $^{13}$ and a mean LOS of 14.9 days in hospital with a standard deviation (SD) of 10.2 days. A sample size of 240 patients (120 per group) was found to be necessary to detect this difference with a power of $80 \%$ and an alpha error of 0.05 .

An intention-to-treat analysis of the primary study outcomes was performed for all randomised patients. Furthermore, primary and secondary outcome parameters were measured for the evaluable study population. A Student's $t$ test was used to compare continuous data; differences in LOS were calculated with the Mann-Whitney test. A $\chi^{2}$ test was used to compare categorical data between the two treatment groups. Results were expressed as mean (SD) values. Differences in outcome between the two treatment groups were calculated with 95\% two-sided confidence intervals (95\% CI). A p value of $<0.05$ was considered significant. Data were analysed using SPSS Version 11.5 for Windows (Chicago, USA).
Table 2 Baseline characteristics of study patients

\begin{tabular}{|c|c|c|}
\hline Characteristics & $\begin{array}{l}\text { PDT } \\
(n=134)\end{array}$ & $\begin{array}{l}\text { EAT } \\
(n=128)\end{array}$ \\
\hline Mean (SD) age (years) & $62.0(18.5)$ & $66.7(17.2) \dagger$ \\
\hline \multicolumn{3}{|l|}{ Sex, $n(\%)$} \\
\hline Male & $74(55)$ & $67(52)$ \\
\hline Female & $60(45)$ & $61(48)$ \\
\hline Nursing home residents, $\mathrm{n}(\%)$ & $3(2)$ & $3(2)$ \\
\hline Outpatient antibiotic treatment, $n(\%)$ & $39(29)$ & $29(23)$ \\
\hline \multicolumn{3}{|c|}{ Co-morbidity, n (\%) } \\
\hline COPD & $47(35)$ & $49(38)$ \\
\hline Asthma & $10(7)$ & $13(10)$ \\
\hline Congestive heart failure & $9(7)$ & $12(9)$ \\
\hline Ischaemic heart disease & $6(4)$ & $11(9)$ \\
\hline Neurological disorder & $13(10)$ & $11(9)$ \\
\hline Liver disease & $2(1)$ & $1(1)$ \\
\hline Chronic renal disease & 0 & $4(3)$ \\
\hline Diabetes mellitus & $14(10)$ & $12(9)$ \\
\hline \multicolumn{3}{|l|}{ Smoking, n (\%) } \\
\hline$\geqslant 10$ cigarettes/day & 25 (19) & 35 (27) \\
\hline$<10$ cigarettes/day & $8(6)$ & $6(5)$ \\
\hline Ex-smoker & $46(34)$ & $42(32)$ \\
\hline Non-smoker & $54(40)$ & 45 (35) \\
\hline Alcohol intake, $\mathrm{n}(\%)$ & & \\
\hline$>3$ units/day & $5(4)$ & $7(5)$ \\
\hline \multicolumn{3}{|l|}{ Pneumonia severity index, $\mathrm{n}(\%)$} \\
\hline Risk class 1 & $15(11)$ & $8(6)$ \\
\hline Risk class 2 & $35(26)$ & 24 (19) \\
\hline Risk class 3 & $31(23)$ & $33(26)$ \\
\hline Risk class 4 & 44 (33) & $50(39)$ \\
\hline Risk class 5 & $9(7)$ & $13(10)$ \\
\hline
\end{tabular}

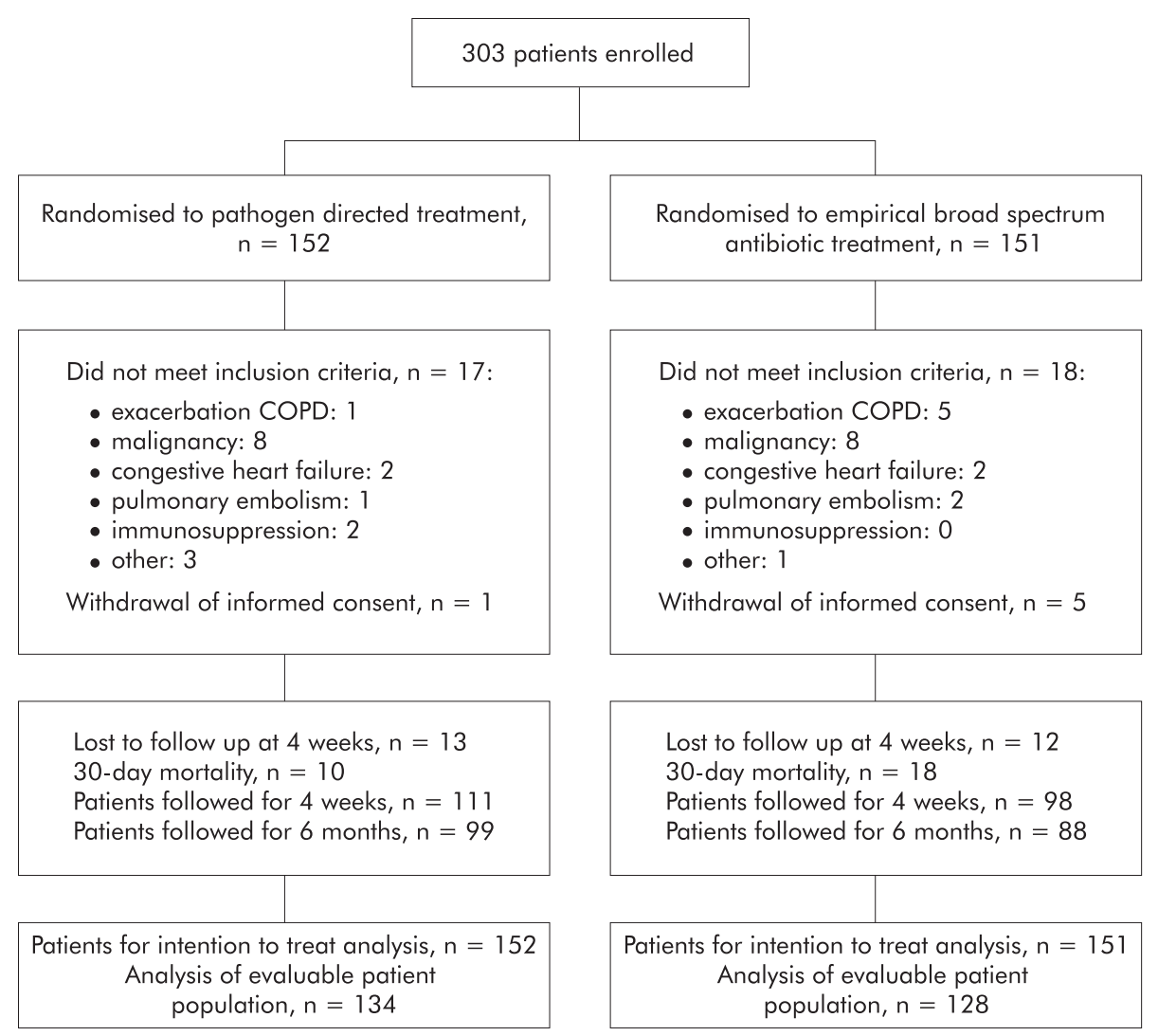

Figure 1 Randomisation to treatment, withdrawal of informed consent, and follow up among 303 patients with community acquired pneumonia. 


\section{RESULTS}

\section{Baseline characteristics}

Three hundred and three patients were included in the study; all were hospitalised. Thirty five patients were initially misdiagnosed and six withdrew consent and were subsequently excluded from the study. The results from 262 patients were evaluable for analysis; of these, 134 were assigned to the PDT group and 128 to the EAT group (fig l). Patients in the PDT group were significantly younger than those in the EAT group (mean age 62.0 years and 66.7 years, respectively; $\mathrm{p}=0.03)$. Eighty two patients $(31 \%)$ stratified in risk class I and II were hospitalised; significantly more in the PDT group $(\mathrm{n}=50(37 \%))$ than in the EAT group $(\mathrm{n}=32$ $(25 \%) ; \mathrm{p}=0.03$, table 2$)$.

\section{Aetiology}

Overall, 196 microorganisms were identified. In 84 patients $(63 \%)$ in the PDT group treatment could be directed at a presumptive or definite microbial diagnosis. A pathogen could not be identified in 50 patients $(37 \%)$ in the PDT group and $59(46 \%)$ in the EAT group. The most common pathogen in both groups was $S$ pneumoniae ( $\mathrm{n}=92$ patients $(35 \%))$, followed by $M$ pneumoniae $(\mathrm{n}=23(9 \%))$ and $H$ influenzae $(\mathrm{n}=19(7 \%))($ table 3$)$. No penicillin or macrolide resistant pneumococcal strains were identified. In 18 of the 22 ICU patients $(82 \%)$ a causative pathogen was identified. The microorganism most frequently identified in this population was $S$ pneumoniae $(\mathrm{n}=9(41 \%))$, followed by L pneumophila $(n=6(27 \%)$, data not shown). Overall, 157 patients $(60 \%)$ produced a sputum specimen; in 44 samples (28\%) a Gram stain of adequate quality was present. In the PDT group 85 patients $(64 \%)$ produced a sputum sample, of which 25 (29\%) were adequate specimens.

\section{Length of stay in hospital, clinical failure, 30 day mortality}

In the intention-to-treat and evaluable study population, no significant difference in LOS was detected between the two treatment groups. In the intention-to-treat population, patients in the PDT group remained in hospital for an average of 14.3 days compared with 13.2 days for the EAT group (95\% CI -1.5 to $3.7 ; \mathrm{p}=0.75)$. In the evaluable patient population, LOS in the PDT and EAT groups was 13.7 days and 12.8 days, respectively $(95 \%$ CI -1.9 to $3.7 ; \mathrm{p}=0.63$; table 4).

In neither the intention-to-treat population nor the evaluable patient population was any significant difference found in clinical failures between the two treatment groups (95\% CI -0.1 to $0.1 ; p=0.66$ and $95 \%$ CI -0.1 to 0.1 ; $\mathrm{p}=0.74$, respectively). In the evaluable population, 26 in the PDT group (19\%) and 27 in the EAT group (21\%) suffered a clinical failure on antibiotic treatment (table 4). In the PDT group, clinical failure occurred in 15 of 62 patients (24\%) who were treated according to rapidly obtained microbial results, and in 11 of 72 patients (15\%) who were treated according to a syndromic approach $(p=0.19$; see fig $S 1$ available on the Thorax website at http://www.thoraxjnl.com/supplemental). In the EAT group, mortality after inadequate treatment occurred in two patients infected with $S$ aureus and in two other patients no pathogen could be identified (table 4).

No significant difference in mortality was seen between the two treatment groups in the intention-to-treat population or

\begin{tabular}{|c|c|c|c|}
\hline Aetiology & PDT $(n=134)$ & EAT $(n=128)$ & Total $(n=262)$ \\
\hline Streptococcus pneumoniae, $\mathrm{n}(\%)$ & $49(37)$ & $43(34)$ & $92(35)$ \\
\hline Definitive & 16 & 15 & 31 \\
\hline Presumptive & 33 & 28 & 61 \\
\hline \multicolumn{4}{|l|}{ Mycoplasma pneumoniae, n (\%) } \\
\hline Definitive & $13(10)$ & $10(8)$ & $23(9)$ \\
\hline Haemophilus influenzae, $\mathrm{n}(\%)$ & $13(10)$ & $6(5)$ & 19 (7) \\
\hline Definitive & 0 & 3 & 3 \\
\hline Presumptive & 13 & 3 & 16 \\
\hline \multicolumn{4}{|l|}{ Legionella pneumophila*, n (\%) } \\
\hline Definitive & $7(5)$ & $7(5)$ & $14(5)$ \\
\hline GNEB $+\mathrm{n}(\%)$ & $2(1)$ & 9 (7) & $11(4)$ \\
\hline Definitive & 0 & 2 & 2 \\
\hline Presumptive & 2 & 7 & 9 \\
\hline Staphylococcus aureus, n (\%) & $6(4)$ & $4(3)$ & $10(4)$ \\
\hline Definitive & 2 & 4 & 6 \\
\hline Presumptive & 4 & 0 & 4 \\
\hline \multicolumn{4}{|l|}{ Moraxella catarrhalis, n (\%) } \\
\hline Presumptive & $7(5)$ & 0 & $7(3)$ \\
\hline Viral infection, $n(\%)$ & & & \\
\hline \multirow{2}{*}{\multicolumn{4}{|c|}{$\begin{array}{l}\text { Definitive } \\
\text { Chlamydia pneumoniae, } n \text { (\%) }\end{array}$}} \\
\hline & & & \\
\hline Definitive & $1(<1)$ & 0 & $1(<1)$ \\
\hline Otherf, $\mathrm{n}(\%)$ & $4(3)$ & $5(4)$ & $9(3)$ \\
\hline Definitive & 0 & 2 & 2 \\
\hline Presumptive & 4 & 3 & 7 \\
\hline Mixed infection $\%, \mathrm{n}(\%)$ & $7(5)$ & $10(8)$ & $17(6)$ \\
\hline Unknown, n (\%) & 50 (37) & $59(46)$ & $109(42)$ \\
\hline
\end{tabular}

PDT, pathogen directed treatment group; EAT, empirical antibiotic treatment group. *Nine cases of Legionnaire's disease (six in the PDT group and three in the EAT group) were the result of a visit to a flower exhibition in Bovenkarspel, the Netherlands.

†Gram negative Enterobacteriaceae: PDT: $E$ coli $(2 \times)$. EAT, definitive diagnoses: $E$ coli $(1 \times)$, Enterobacter cloacae $(1 \times)$. EAT, presumptive diagnoses: E coli $(1 \times)$, Enterobacter aeruginosa $(1 \times)$, Klebsiella pneumoniae $(1 \times)$, Citrobacter freundii $(1 \times)$, Klebsiellla oxytoca $(1 \times)$, Morganella morgagnii $(1 \times)$, E cloacae $(1 \times)$. †PDT: Haemophilus parainfluenzae $(2 \times)$, Pseudomonas aeruginosa $(1 \times), \alpha$-haemolytic streptococcus $(1 \times)$. EAT, definitive diagnoses: Arcanobacterium haemolyticum $(1 \times)$ and streptococcus group $C(1 \times)$. EAT, presumptive diagnoses: $P$ aeruginosa $(1 \times)$, streptococcus group $B(1 \times)$, Staphylococcus epidermidis $(1 \times)$.

- Mixed infection consisted of a typical bacterial pathogen and a definitive atypical bacterial or viral pathogen. 
Table 4 Clinical efficacy of treatment in the two treatment groups

\begin{tabular}{|c|c|c|c|c|c|}
\hline Outcome & PDT & EAT & $95 \% \mathrm{Cl}$ & p value & Odds ratio $(95 \% \mathrm{Cl})$ \\
\hline Intention-to-treat population, $\mathrm{n}$ & 152 & 151 & & & \\
\hline LOS, mean days $\left(\mathrm{Cl}^{*}\right)$ & $14.3(12.2$ to 16.4$)$ & 13.2 (11.7 to 14.7$)$ & -1.5 to 3.7 & 0.75 & \\
\hline Clinical failure, $\mathrm{n}(\%)$ & $32(21)$ & $35(23)$ & $-12 \%$ to $7 \%$ & 0.66 & $1.13(0.66$ to 1.95$)$ \\
\hline Mortality, n (\%) & $12(8)$ & $22(15)$ & $-14 \%$ to $1 \%$ & 0.07 & $1.99(0.95$ to 4.18$)$ \\
\hline Evaluable population, $\mathrm{n}$ & 134 & 128 & & & \\
\hline LOS, mean days (CI) & 13.7 (11.5 to 15.9$)$ & $12.8(11.1$ to 14.5$)$ & -1.9 to 3.7 & 0.63 & \\
\hline Clinical failure, $n(\%)$ & $26(19)$ & $27(21)$ & $-12 \%$ to $8 \%$ & 0.74 & 1.11 (0.61 to 2.03$)$ \\
\hline Mortality, n (\%) & $10(7)$ & $18(14)$ & $-14 \%$ to $1 \%$ & 0.09 & 2.03 (0.90 to 4.58$)$ \\
\hline Early failuret, n (\%) & 17 (13) & $15(12)$ & $-8 \%$ to $9 \%$ & 0.81 & \\
\hline \multicolumn{6}{|l|}{ Adequate antibiotic } \\
\hline Treatment, n (\%) & $9(7)$ & $10(8)$ & $-7 \%$ to $5 \%$ & 0.73 & \\
\hline Mortality, n (\%) & $4(3)$ & $6(5)$ & $-6 \%$ to $3 \%$ & 0.47 & \\
\hline \multicolumn{6}{|l|}{ Inadequate antibiotic } \\
\hline Treatment, n (\%) & $8(6)$ & $5(4)$ & $-3 \%$ to $7 \%$ & 0.44 & \\
\hline Mortality, n (\%) & $2(1)$ & $4(3)$ & $-6 \%$ to $2 \%$ & 0.23 & \\
\hline Late failuref, n (\%) & $9(7)$ & $12(9)$ & $-9 \%$ to $4 \%$ & 0.43 & \\
\hline Mortality, n (\%) & $4(3)$ & $8(6)$ & $-8 \%$ to $2 \%$ & 0.21 & \\
\hline ICU admission, $\mathrm{n}(\%)$ & $11(8)$ & $11(9)$ & $-7 \%$ to $6 \%$ & 0.91 & \\
\hline Mortality, n (\%) & $5(45)$ & $10(91)$ & $-84 \%$ to $-7 \%$ & 0.02 & \\
\hline Adequate treatment, $\mathrm{n}(\%)$ & $4(80)$ & $8(80)$ & $-50 \%$ to $50 \%$ & 1 & \\
\hline Inadequate treatment, n (\%) & $1(20)$ & $2(20)$ & $-50 \%$ to $50 \%$ & 1 & \\
\hline Clinical failure, $\mathrm{n}(\%)$ & $8(73)$ & $11(100)$ & $-59 \%$ to $4 \%$ & 0.08 & \\
\hline
\end{tabular}

PDT, pathogen directed treatment group; EAT, empirical antibiotic treatment group; $95 \% \mathrm{Cl}, 95 \%$ confidence interval for difference; LOS, length of stay; ICU, intensive care unit.

*95\% confidence interval for mean.

†Failure within 72 hours of starting antibiotic treatment and thereafter.

†Failure after 72 hours of treatment.

in the evaluable patient population ( $95 \%$ CI -0.14 to 0.01 ; $\mathrm{p}=0.07$ and $95 \% \mathrm{CI}-0.14$ to $0.01 ; \mathrm{p}=0.09$, respectively; table 4$)$. In the evaluable population, 10 patients $(7 \%)$ in the PDT group died compared with 18 (14\%) in the EAT group. Eleven patients in each group were referred to the ICU. From this subgroup, significantly more EAT patients than PDT patients died (10 (91\%) v 5 (45\%); $95 \%$ CI -0.84 to -0.07 ; $\mathrm{p}=0.02)$. The real failures in the ICU group consisted of one PDT patient and two EAT patients who were treated inadequately and subsequently died (see table S1 available on the Thorax website at http://www.thoraxjnl.com/supplemental). A significant difference $(p=0.03)$ in mortality was observed in the PDT population between 72 patients (54\%) who were treated according to a syndromic approach and 62 (46\%) who received treatment directed at a pathogen suspected from rapid routine microbiological investigations (mortality rates $3 \%(\mathrm{n}=2) v 13 \%(\mathrm{n}=8)$, respectively; see fig S1 available on the Thorax website at http://horaxjnl.com/ supplemental).
Patients in both groups were treated for a mean period of 5 days with IV antibiotics and completed their treatment course after 10.8 days in the PDT group and 9.9 days in the EAT group. Patients were afebrile by day 3 of treatment (table 5).

\section{Antibiotic choice and adverse events}

All patients except one in the PDT group were started on IV monotherapy. Penicillin G was used most frequently $(n=54$ $(40 \%))$, followed by amoxicillin-clavulanate $(n=47(35 \%))$, erythromycin $(n=16(12 \%))$, amoxicillin $(n=9(7 \%))$, cefuroxime $(\mathrm{n}=3(2 \%))$, and other $(\mathrm{n}=5(4 \%))$. Antibiotic treatment was adapted in 25 patients $(19 \%)$ in the PDT group to microbial culture results. Ten patients $(7 \%)$ received two antibiotics during hospitalisation because of adaptation to culture results or clinical failure on monotherapy. Patients in the EAT group initially received a combination of erythromycin and amoxicillin-clavulanate $(\mathrm{n}=112(88 \%))$, erythromycin + cefuroxime $(n=8(6 \%))$, doxycycline + amoxicillin-clavulanate

Table 5 Secondary outcome parameters

\begin{tabular}{|c|c|c|c|c|}
\hline Variables & PDT & EAT & $95 \% \mathrm{Cl}$ & $p$ value \\
\hline Mean (SD) ABiv (days) & $5.4(4.3)$ & $5.1(3.8)$ & -0.6 to 1.4 & 0.47 \\
\hline Mean (SD) AB (days) & $10.8(5.5)$ & $9.9(5.0)$ & -0.4 to 2.1 & 0.19 \\
\hline Mean (SD) time to resolution of fever (days) & $2.9(2.7)$ & $2.5(2.5)$ & -0.3 to 1.0 & 0.24 \\
\hline Adverse events, no of patients (\%) & $23(17)^{*}$ & $77(60) \dagger$ & $-50 \%$ to $-30 \%$ & $<0.001$ \\
\hline \multicolumn{5}{|l|}{ Mean (SD) SF-36 score [no of patients] } \\
\hline Hospitalisation & $60.2(23.4)[n=72]$ & $58.3(23.3)[n=59]$ & -6.2 to 9.9 & 0.65 \\
\hline 30 day follow up & $59.5(21.5)[n=59]$ & $57.3(20.5)[n=47]$ & -6.0 to 10.3 & 0.60 \\
\hline 90 day follow up & 66.7 (22.9) $[n=50]$ & $67.2(30.1)[n=35]$ & -11.9 to 10.9 & 0.93 \\
\hline 180 day follow up & $79.3(22.4)[n=31]$ & $64.1(20.1)[n=22]$ & +3.3 to 27.3 & 0.01 \\
\hline \multicolumn{5}{|l|}{ Mean (SD) SSC score [no of patients] } \\
\hline Admission & $49.8(16.2)[n=110]$ & $47.0(18.3)[n=95]$ & -1.9 to 7.6 & 0.24 \\
\hline Day 7 of treatment & $21.6(14.2)[n=92]$ & $19.3(12.5)[n=70]$ & -1.9 to 6.5 & 0.28 \\
\hline 30 day follow up & $13.0(10.1)[n=107]$ & $11.2(7.8)[n=95]$ & -0.7 to 4.4 & 0.15 \\
\hline 90 day follow up & 9.1 (9.7) $[n=102]$ & $9.6(9.6)[n=89]$ & -3.3 to 2.2 & 0.69 \\
\hline 180 day follow up & $7.4(9.8)[n=94]$ & $9.9(10.8)[n=85]$ & -5.6 to 0.5 & 0.10 \\
\hline
\end{tabular}

PDT, pathogen directed treatment group; EAT, empirical antibiotic treatment group; $95 \% \mathrm{Cl}, 95 \%$ confidence interval for difference; $\mathrm{ABiv}$, duration of intravenous antibiotic treatment; $A B$, total duration of antibiotic treatment; SF-36, quality of life questionnaire; SSC, symptom severity score.

*Adverse events in PDT group were: gastrointestinal disturbances $(n=9)$, phlebitis $(n=8)$, reversible deafness $(n=5)$, reversible hair loss $(n=5)$, urticaria $(n=2)$. †Adverse events in EAT group were: gastrointestinal disturbances $(n=42)$, phlebitis $(n=38)$, reversible deafness $(n=14)$, reversible hair loss $(n=10)$, urticaria $(\mathrm{n}=2)$; some patients had more than one adverse event. 
$(\mathrm{n}=1(0.8 \%))$ or erythromycin + ceftazidime $(\mathrm{n}=7(5 \%))$ as IV antibiotic treatment (data not shown). Four patients referred to the ICU during treatment initially received erythromycin and amoxicillin-clavulanate, which was later replaced by erythromycin and ceftazidime.

Significantly more adverse events were encountered in the EAT group than in the PDT group ( 77 patients $(60 \%) v 23$ patients (17\%), 95\% CI -0.5 to $-0.3 ; p<0.001$, table 5$)$. The adverse events did not have any significant clinical influence on outcome parameters such as LOS, treatment failure, and mortality.

\section{DISCUSSION}

In this prospective randomised study no significant difference in outcome between a pathogen directed approach and an empirical strategy with broad spectrum antibiotics, as proposed by the ATS guidelines of 1993, was found in adult hospitalised patients with CAP. Only adverse events, although not clinically significant, occurred significantly more often in the EAT group than in the PDT group $(p<0.001)$. Neither therapeutic regimen had any significant effect on LOS. It is known that there is a difference in LOS of patients with CAP between Europe and the USA. ${ }^{14-17}$ This may be explained by regional differences such as more economic pressure in the USA. Treatment of co-morbid illness, social factors and a lack of immediate outpatient supportive care facilities prolonged hospital stay in several cases in our study. These have also been observed in two other studies. ${ }^{15} 18$ It should be emphasised that, in this study, no criteria for discharge were given to the treating physician. The decision to discharge a patient from hospital was based on clinical judgement. However, we are of the opinion that a closer evaluation of hospital discharge criteria would have led to a reduction in LOS. Some authors have suggested that, in certain circumstances, a patient could be discharged directly after changing from IV to oral treatment. ${ }^{19-21}$

With regard to our secondary outcome parameters, both treatment strategies were successful in the evaluable patient population with only $6 \%$ and $4 \%$ early failures after inappropriate antibiotic prescriptions, as a result of which two (1\%) and four (3\%) patients died in the PDT and EAT groups, respectively. Patients in the EAT group were significantly older than those in the PDT group $(p=0.03)$, and this could have influenced the results as age is a known risk factor for mortality. ${ }^{10}$ The presence of significantly more low risk patients in the PDT population $(p=0.03)$ did not have a significant influence on the outcome parameters.

In a subanalysis, a significant difference $(p=0.02)$ was found in mortality between PDT and EAT patients who had been admitted to the ICU (45\% v 91\%). It is difficult to explain the high mortality rate in EAT patients. One possible explanation is that, as the study was not designed to detect a difference in mortality in ICU patients, the relatively low number of ICU patients made it difficult to establish a firm conclusion about this population. Seven EAT patients (64\%) received a combination of ceftazidime and erythromycin and four $(36 \%)$ initially received a combination of amoxicillinclavulanate and erythromycin which was later switched to ceftazidime and erythromycin when they were referred to the ICU. Gleason $e^{2} a^{22}$ found a higher 30 day mortality rate after using a $\beta$-lactam/ $\beta$-lactamase inhibitor plus macrolide compared with other antibiotics. In another study cephalosporin treatment was associated with a worse prognosis than treatment with penicillin. ${ }^{23}$ In the ATS guidelines of 2001 a clear distinction has been made between treatment of ICU patients with and without risk factors for $P$ aeruginosa infection. Ceftriaxone and cefotaxime are currently the preferred cephalosporins for treating ICU patients without risk factors for $P$ aeruginosa infection, instead of the other third generation cephalosporins with anti-Pseudomonas activity such as ceftazidime which were recommended in the ATS guidelines of $1993 .{ }^{3}{ }^{4}$ When analysing each ICU patient individually, it is debatable whether the two deaths caused by inappropriate treatment in the EAT group could have been avoided by a pathogen directed approach. Six patients in the EAT group died after adequate antibiotic treatment and their deaths could not be explained by the use of any particular antibiotic regimen. Furthermore, four deaths in the EAT group occurred after a late failure caused by non-pneumonia related ilnesses. Some authors have remarked that death and clinical failure sometimes cannot be explained by the use of antibiotics alone. ${ }^{24} 25$ In some patients death is inevitable despite appropriate treatment. Austrian and Gold ${ }^{26}$ described this as the moment when patients have passed the physiological "point of no return".

An argument against a pathogen directed approach is that atypical bacterial pathogens such as $L$ pneumophila, $M$ pneumoniae and $C$ pneumoniae, for which the prevalence ranges from $8 \%$ to $63 \%,{ }^{27-30}$ cannot be identified by conventional microbiological methods during the first days of treatment. The results of this study showed that, with the exception of L pneumophila, the inability to identify these atypical microorganisms in the PDT group did not result in a significantly higher mortality or clinical failure rate than in the EAT group, an observation which has also been reported by others. ${ }^{31-33}$ To increase detection of $L$ pneumophila infection, the urinary antigen test for this pathogen should be used as a routine diagnostic investigation in ICU patients since there is a strong association between legionella infection and the need for admission to an ICU. ${ }^{34}$

In our study, when microbiological results were not available in the PDT group, we used a syndromic approach consisting of a combination of symptoms obtained from the literature suggestive of certain pathogens to select the initial antibiotic. Surprisingly, treatment according to a syndromic approach was associated with a lower clinical failure and mortality rate than treatment directed at results obtained by microbial investigation $(15 \% \vee 24 \%(\mathrm{p}=0.19)$ and $3 \% \vee 13 \%$ $(p=0.03)$, respectively). Seventy percent of the patients in whom a pathogen was identified after receiving treatment according to a syndromic approach had initially received adequate treatment. However, it is known from the literature that no single features are sufficiently reliable to enable the aetiology to be guessed accurately. ${ }^{29} 35$

Adverse events occurred significantly more often in the EAT group than in the PDT group, although they did not have any significant clinical influence on the outcome parameters such as LOS, treatment failure, or mortality. It is very likely that the gastrointestinal disturbances were caused by the use of IV erythromycin. Furthermore, after changing from erythromycin IV to oral azithromycin, the sensation of deafness experienced by our study population improved.

The ATS guidelines have recently been revised and adapted to include new developments in the treatment of CAP. ${ }^{4}$ The 2001 ATS guidelines are essentially the same as the 1993 guidelines for hospitalised patients with cardiopulmonary disease. For ICU patients, management depends on the presence of risk factors for $P$ aeruginosa infection. New therapeutic options have been proposed in which the use of the new fluoroquinolones are becoming more prominent.

In this study no penicillin resistant pneumococcal strains were identified. In the Netherlands antibiotic usage is limited, ${ }^{36}$ which is reflected by a low prevalence of penicilin resistant pneumococcal strains (1.5\%) in 2002 (European Antimicrobial Resistance Surveillance System; www.earss.rivm.nl). In the USA, community acquired $S$ pneumoniae isolates are generally more resistant to penicillin $(16.7 \%) .{ }^{37}$

We recognise that this study has some limitations. Firstly, for logistical reasons it was not possible to perform a double 
blind, randomised study. Secondly, the study was powered according to LOS and not according to mortality. The reason for the choice of LOS was because of the lack of studies that investigated the effectiveness of implementing the ATS guidelines at the time we completed the study protocol. The only study on which we could base our power analysis was one which correlated the implementation of the ATS guidelines with LOS. ${ }^{13}$ We realised that LOS would be influenced by non-pneumonia factors such as treatment of co-morbidity or social circumstances. Given the observed mortality rates in our study, 322 patients in each group (644 patients in total) would be needed to show a significant difference in mortality between the two groups. For practical reasons this number of patients could not be included within the established period in which we performed our study. Thirdly, we implemented antibiotic treatment according to the 1993 ATS guidelines for the empirically treated group. New guidelines were presented in 2001 but are not essentially different for hospitalised patients with the exception of ICU patients. Another limitation is the admission of low risk patients (risk class I-II). In our study the presence of other active co-morbidities and the clinical judgement that the pneumonia was worse than the PSI score indicated influenced the decision to admit a low risk patient.

In conclusion, we have attempted to compare two different treatment strategies in the management of CAP. Our results show that an empirical approach with broad spectrum antibiotics has comparable efficacy to a pathogen directed approach.

\section{ACKNOWLEDGEMENTS}

The authors thank Mr T van der Ploeg for statistical advice and Mrs Y Holloway for her assistance in editing this manuscript.

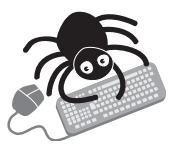

Figure S1 and table S1 are available online at http:// www.thoraxjnl.com/supplemental.

\section{Authors' affiliations}

M M van der Eerden, C S de Graaff, W G Boersma, Department of Pulmonary Diseases, Medical Centre Alkmaar, Alkmaar, the Netherlands

F Vlaspolder, T Groot, Laboratory for Medical Microbiology, Medical Centre Alkmaar, Alkmaar, the Netherlands

W Bronsveld, Department of Internal Medicine, Medical Centre Alkmaar, Alkmaar, the Netherlands

H M Jansen, Department of Pulmonary Diseases, Academic Medical Centre, Amsterdam, the Netherlands

Part of this work was presented at the 98th International Conference of the American Thoracic Society, Atlanta, USA, 17-22 May 2002.

\section{REFERENCES}

1 Fine MJ, Smith MA, Carson CA, et al. Prognosis and outcomes of patients with community-acquired pneumonia. A meta-analysis. JAMA 1996;275:134-41.

2 British Thoracic Society Standards of Care Comittee. BTS Guidelines for the management of community-acquired pneumonia in adults. Thorax 2001;56(Suppl IV):iv1-64.

3 Niederman MS, Bass JB Jr, Campbell GD, et al. Guidelines for the initial management of adults with community-acquired pneumonia: diagnosis, assessment of severity, and initial antimicrobial therapy. Am Rev Respir Dis 1993; 148:1418-26.

4 Niederman MS, Mandell LA, Anzueto A, et al. Guidelines for the management of adults with community-acquired pneumonia. Diagnosis, assessment of severity, antimicrobial therapy, and prevention. Am J Respir Crit Care Med $2001 ; 163: 1730-54$

5 Bartlett JG, Dowell SF, Mandell LA, et al. Practice guidelines for the management of community-acquired pneumonia in adults. Clin Infect Dis 2000;31:347-82.

6 Wise R, Hart T, Cars O, et al. Antimicrobial resistance. BMJ 1998;317:609-10.

7 Gould IM. A review of the role of antibiotic policies in the control of antibiotic resistance. J Antimicrob Chemother 1999;43:459-65.
8 San Pedro GS, Campbell GD Jr. Limitations of diagnostic testing in the initial management of patients with community-acquired pneumonia. Semin Respir Infect 1997;12:300-7

9 Sanyal S, Smith PR, Saha AC, et al. Initial microbiologic studies did not affect outcome in adults hospitalized with community-acquired pneumonia. Am J Respir Crit Care Med 1999; 160:346-8.

10 Fine MJ, Auble TE, Yealy DM, et al. A prediction rule to identify low-risk patients with community-acquired pneumonia. N Engl J Med 1997:336:243-50

11 Ware JE Jr, Sherbourne CD. The MOS 36-item short-form health survey (SF36). I. Conceptual framework and item selection. Med Care 1992;30:473-83

12 Metlay JP, Fine MJ, Schulz R, et al. Measuring symptomatic and functional recovery in patients with community-acquired pneumonia. J Gen Intern Med 1997:12:423-30.

13 Webster S, Dodek P. Implementation of the American Thoracic Society (ATS) guideline for the initial management of community-acquired pneumonia improves appropriateness of antibiotic use. Am J Respir Crit Care Med 1997:155:A234.

14 Ramirez JA, Vargas S, Ritter GW, et al. Early switch from intravenous to oral antibiotics and early hospital discharge: a prospective observational study of 200 consecutive patients with community-acquired pneumonia. Arch Intern Med 1999; 159:2449-54.

15 Rosón B, Carratalà J, Dorca J, et al. Etiology, reasons for hospitalization, risk classes, and outcomes of community-acquired pneumonia in patients hospitalized on the basis of conventional admission criteria. Clin Infect Dis 2001;33:158-65.

16 Menéndez $R$, Ferrrando $D$, Vallés $J M$, et al. Initial risk class and length of hospital stay in community-acquired pneumonia. Eur Respir $J$ $2001 ; 18: 151-6$.

17 Eron LJ, Passos S. Early discharge of infected patients through appropriate antibiotic use. Arch Intern Med 2001;161:61-5

18 Fine MJ, Medsger AR, Stone RA, et al. The hospital discharge decision for patients with community-acquired pneumonia. Results from the Pneumonia Patient Outcomes Research Team Cohort Study. Arch Intern Med 1997; 157:47-56

19 Boyter AC, Stephen J, Fegan PG, et al. Why do patients with infection remain in hospital once changed to oral antibiotics? J Antimicrob Chemother 1997;39:286-8.

20 Farber BF, Cooper DJ. Observing patients after antibiotics are discontinued. N Engl J Med 1995;333:1083-4.

21 Dunn AS, Peterson KL, Schechter CB, et al. The utility of an in-hospital observation period after discontinuing intravenous antibiotics. Am J Med 1999;106:6-10.

22 Gleason PP, Meehan TP, Fine JM, et al. Associations between initial antimicrobial therapy and medical outcomes for hospitalized elderly patients with pneumonia. Arch Intern Med 1999;159:2562-72.

23 Kalin M, Örtqvist A, Almela $M$, et al. Prospective study of prognostic factors in community-acquired bacteremic pneumococcal disease in 5 countries. $J$ Infect Dis 2000;182:840-7

24 Hook EW, Horton CA, Schaberg DR. Failure of intensive care unit support to influence mortality from pneumococcal bacteremia. JAMA 1983;249: 1055-7.

25 Knaus WA. Changing the cause of death. JAMA 1983;249:1059-60.

26 Austrian R, Gold J. Pneumococcal bacteremia with especial reference to bacteremic pneumococcal pneumonia. Ann Intern Med 1964:60:759-76.

27 Lieberman D, Schlaeffer F, Boldur I, et al. Multiple pathogens in adult patients admitted with community-acquired pneumonia: a one year prospective study of 346 consecutive patients. Thorax 1996;51:179-84.

28 Lim WS, Macfarlane JT, Boswell TC, et al. Study of community acquired pneumonia aetiology (SCAPA) in adults admitted to hospital: implications for management guidelines. Thorax 2001;56:296-301.

29 Fang GD, Fine $M$, Orloff J, et al. New and emerging etiologies for communityacquired pneumonia with implications for therapy. A prospective multicenter study of 359 cases. Medicine 1990;69:307-16.

30 Mundy LM, Auwaerter PG, Oldach D, et al. Community-acquired pneumonia: impact of immune status. Am J Respir Crit Care Med 1995; 152:1309-15.

31 File TM Jr, Segreti J, Dunbar L, et al. A multicenter, randomized study comparing the efficacy and safety of intravenous and/or oral levofloxacin versus ceftriaxone and/or cefuroxime axetil in treatment of adults with community-acquired pneumonia. Antimicrob Agents Chemother 1997; 41:1965-72.

32 Rosón B, Carratalà J, Verdaguer R, et al. Prospective study of the usefulness of sputum Gram stain in the initial approach to community-acquired pneumonia requiring hospitalization. Clin Infect Dis 2000;31:869-74.

33 Mundy LM, Oldach D, Auwaerter PG, et al. Implications for macrolide treatment in community-acquired pneumonia. Hopkins CAP Team. Chest 1998;113:1201-6.

34 Stout JE, Yu VL. Legionellosis. N Engl J Med 1997;337:682-7.

35 Farr BM, Kaiser DL, Harrison BDW, et al. Prediction of microbial aetiology at admission to hospital for pneumonia from the presenting clinical features. Thorax 1989;44:1031-5.

36 Van Kasteren ME, Wiinands WJ, Stobberingh EE, et al. Optimization of the antibiotic policy in the Netherlands. II. SWAB guidelines for the antimicrobial therapy of pneumonia in patients at home and as nosocomial infections. Ned Tijdschr Geneeskd 1998;142:952-6.

37 Gordon KA, Biedenbach DJ, Jones RN. Comparison of Streptococcus pneumoniae and Haemophilus influenzae susceptibilities from communityacquired respiratory tract infections and hospitalized patients with pneumonia: five-year results for the SENTRY antimicrobial surveillance program. Diagn Microbiol Infect Dis 2003;46:285-9. 